\title{
Transformation of a mathematical model of gas flow distribution to solve the problems of gas supply system development
}

\author{
Nikolay Ilkevich ${ }^{l}$, Tatyana Dzyubina ${ }^{I, *}$, and Zhanna Kalinina ${ }^{l}$ \\ ${ }^{1}$ Melentiev Energy Systems Institute of Siberian branch of Russian Academy of Science (ESI SB \\ RAS), department of pipeline and hydraulic systems, Lermontov Street 130, Irkutsk, Russia, 664033
}

\begin{abstract}
This paper proposes taking into account new properties of gas supply systems in a mathematical model of flow distribution in comparison with the traditional formulation. The approach suggests introducing an arc coefficient, which allows for changes in the magnitude of gas flow passing along the arc, a vector of an increase in the arc throughput, and lower constraints on the gas flow along the arc. We also propose considering a new economic environment, namely, new criteria for optimizing the flow distribution and setting fictitious gas prices for consumers. These criteria enable us to take account of the priority gas supply to a definite group of consumers. As an example, the calculation of gas flows for the aggregated Unified Gas Supply System (UGSS) for 2030 is considered. This calculation takes into account the arc coefficients and the increase in the throughput of arcs.
\end{abstract}

Keywords: gas supply system, mathematical model of gas flow distribution, arc coefficient, arc gain.

\section{Introduction}

The relevance of the research is due to the growing scale and complexity of gas supply systems (GSS) [1]. The construction of new gas transmission corridors leads to an increase and complication of gas supply systems. Modern mathematical models for studying the development of gas supply should take into account the transformations that reflect the renovation and modernization of gas supply systems [2,3].

In this regard, this paper proposes considering the generalized problem of flow modeling from the viewpoint of analyzing and taking into account the new properties of the gas supply system in comparison with the traditional formulation.

\section{Network analysis methods for gas supply system research}

Network analysis based on graph theory [4] is widely used to solve problems of the gas supply system operation under various conditions. In Russia, researchers started to pay closer

\footnotetext{
*Corresponding author: tvleo@isem.irk.ru
} 
attention to such problems at the end of the 1980s [5-8]. Research teams in other countries also conduct such studies. For example, they investigate the development of the gas supply system of Europe [9, 10], which includes gas supply systems of different countries, and take account of gas supplies from the Russian UGSS [11-15].

The relationships between the GSS facilities are described by a graph $(\bar{R}, \bar{U})$ with a set of network nodes $\bar{R}$ and a set of $\operatorname{arcs} \bar{U} . u \in \bar{U}$ is arc number on the network scheme; $(i, j$ ) stands for an arc with flow direction from node $i$ to node $j,(i, j \in \bar{R})$. If the pipeline allows reverse flow, then two arcs are considered: $(i j)$ and $(j i)$.

Each $\operatorname{arc} u \in \bar{U}$ is assigned the following values: $x_{u}\left(x_{i j}\right)$ - gas flow along the arc; $d_{u}\left(d_{i j}\right)$ throughput of this arc; $c_{u}\left(c_{i j}\right)$ - the price of gas transportation along the arc. Additional nodes $s$ and $t$ are introduced - a common source and sink.

One of the traditional formulations is the minimum cost flow problem, which is written in the form

$$
\begin{aligned}
& \sum_{(i, j) \in U} c_{i j} x_{i j} \rightarrow \min \\
& \sum_{i} x_{i j}-\sum_{i} x_{j i}= \begin{cases}-v, & j=s \\
o, & j \neq s, t \\
w, & j=t\end{cases} \\
& \quad 0 \leq x_{i j} \leq d_{i j},(i, j) \in U,
\end{aligned}
$$

where $v$ is the value of the flux leaving the common source $s$, equal to the sum of flows along the arcs $-\sum_{j} x_{s j}$. From conditions (2), this value is equal to the total flow $w-\sum_{j} x_{i t}$, entering the sink node $t$.

\section{Generalized stream simulation problem}

Used to solve problems of the first level [2], written in the form:

$$
\begin{aligned}
& \sum_{(i, j)}\left(c_{i j} x_{i j}+k_{i j} y_{i j}\right) \rightarrow \min \\
& \sum_{i} \lambda_{i j}-\sum_{i} x_{j i}= \begin{cases}-v, & j=s \\
0, & j \neq s, t \\
w, & j=t\end{cases} \\
& l_{i j} \leq x_{i j} \leq d_{i j}+y_{i j}, \quad(i, j) \in U \\
& 0 \leq y_{i j} \leq g_{i j}, \quad(i, j) \in U
\end{aligned}
$$

Let us consider in more detail the proposals for taking into account the new properties of the gas supply system in comparison with the traditional formulation of the flow distribution problem.

\subsection{Introduction of the arc coefficient}

The arc coefficient $\lambda_{i j}$ (see (5)) allows taking into account the change in the value of the flow $x_{i j}$ passing along the arc. The $\lambda_{i j}$ coefficient takes into account: auxiliary gas consumption or other losses $\left(\lambda_{i j}<1\right)[16,17]$, associated small withdrawals, gas inflows $\left(\lambda_{i j}>1\right)$ from small sources to reduce the size of the modeled system [17]. 


\subsection{Introduction of the vector of capacity gain of arcs}

Introduction of the vector of capacity gain of $\operatorname{arcs} \mathrm{y}=\left(y_{i j}\right)$ and of the vector of constraints on the increase in the capacity $g=\left(g_{i j}\right)$ in the conditions of problem (4), (6), (7).

The commissioning of new capacities is taken into account $[2,16]$. The criterion is the cost function (see (4)). The costs proportional to the flow $\sum_{(i, j)} c_{i j} x_{i j}$ reflect the share of existing costs, while the costs proportional to the increase in capacity $\sum_{(i, j)} k_{i j} y_{i j}$ are the share of the costs of creating new capacity.

\subsection{Introduction of lower constraints on flows along arcs}

The lower constraints $\left(l_{i j}\right)$ (see 6) [2] help to determine the possibility of providing some consumers with gas not lower than a given level of gas consumption, or when it is necessary to use a certain share of the resources of some fields or underground gas storage facilities.

\subsection{The possibility of taking into account new economic conditions}

The problem of choosing a criterion is the most important when taking into account the conditions of the current management. Various groups of criteria are proposed in [16]. None of these criteria are universal, but their variations can reflect different interests $[2,18]$.

\subsection{Setting fictitious prices for gas consumption}

This makes it possible to take into account the priority gas supply to a certain group of consumers [2].

The listed transformations of the mathematical model of gas flow distribution are sequentially implemented in computer systems and programs.

\section{Calculation examples}

Based on the information base on the gas supply systems of the Russian Federation [2] and the energy strategy of Russia [19], we built the UGSS calculation scheme. The quasidynamics of development for the medium term was determined, given gas supplies to the FSU and non-FSU countries [20]. Model conditions are summarized in Table. 1.

Table 1. Introduction of new capacities of the gas transmission system.

\begin{tabular}{|c|c|c|c|c|c|}
\hline \multicolumn{2}{|c|}{ Tie lines } & \multicolumn{3}{c|}{ Capacity, billion $\mathbf{~ m}^{\mathbf{3}}$} & $\operatorname{Arc~coefficient~}\left(\lambda_{i j}\right)$ \\
\hline Initial node & Final node & $\begin{array}{c}\text { fact } \\
\left(d_{i j}\right)\end{array}$ & $\begin{array}{c}\text { new } \\
\left(g_{i j}\right)\end{array}$ & $\begin{array}{c}\text { calculated } \\
\left(y_{i j}\right)\end{array}$ & \\
\hline 100104 & 100130 & 42 & 30 & 30 & 0.996 \\
\hline 100106 & 100101 & & 60 & 24 & 0.999 \\
\hline 100106 & 100109 & & 60 & 25 & 0.983 \\
\hline$\ldots$ & $\ldots$ & $\ldots$ & $\ldots$ & $\ldots$ & $\ldots$ \\
\hline
\end{tabular}


Fig. 1 shows the calculation results for the medium term.

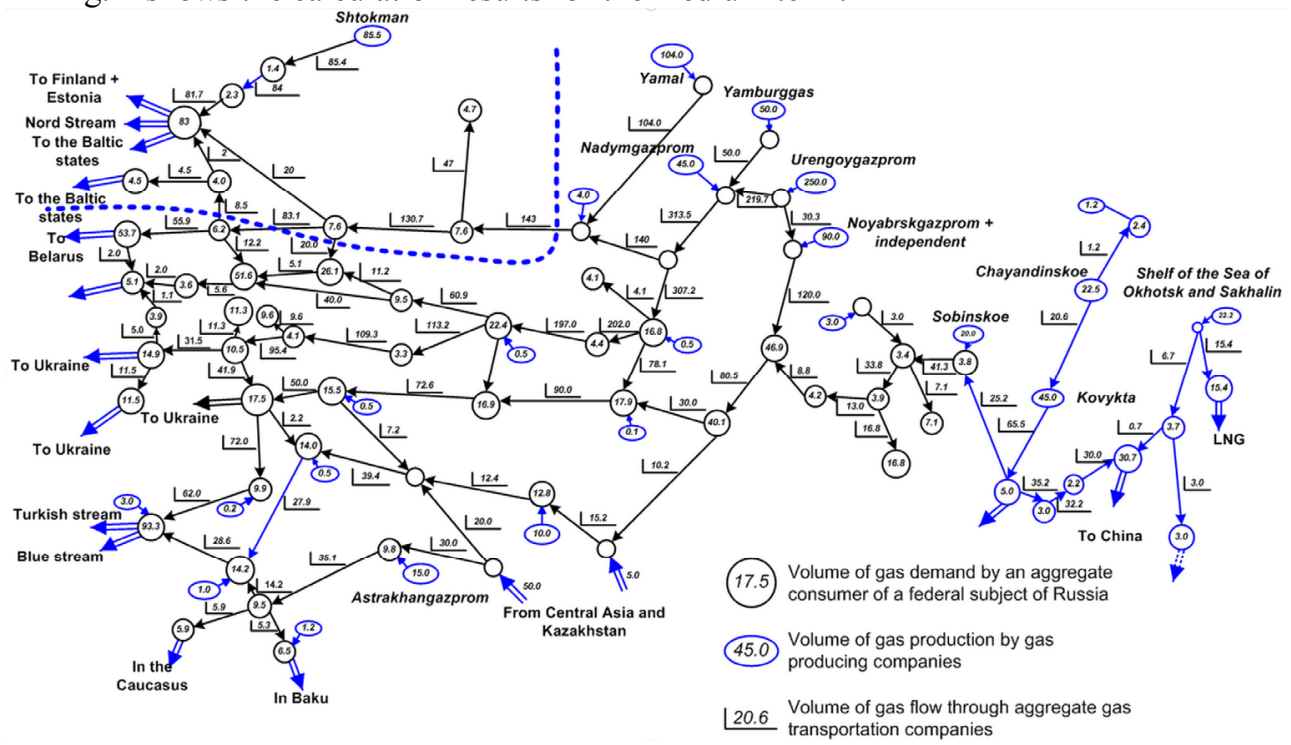

Fig. 1. Gas flows for the aggregated UGSS in the medium term

The Figure and Table show that it is necessary to introduce significant volumes of new capacities of main gas pipelines and compressor capacities in the gas transportation system of the Russian Federation.

The dotted line in Fig. 1 highlights the scheme of gas supply to the Northwestern Federal District. This scheme exemplifies solving the development problems with the help of the flow distribution model at lower levels of consideration.

The model of regulation of seasonal unevenness [2] was used to calculate a detailed gas supply scheme for the Northwestern Federal District (Fig. 2).

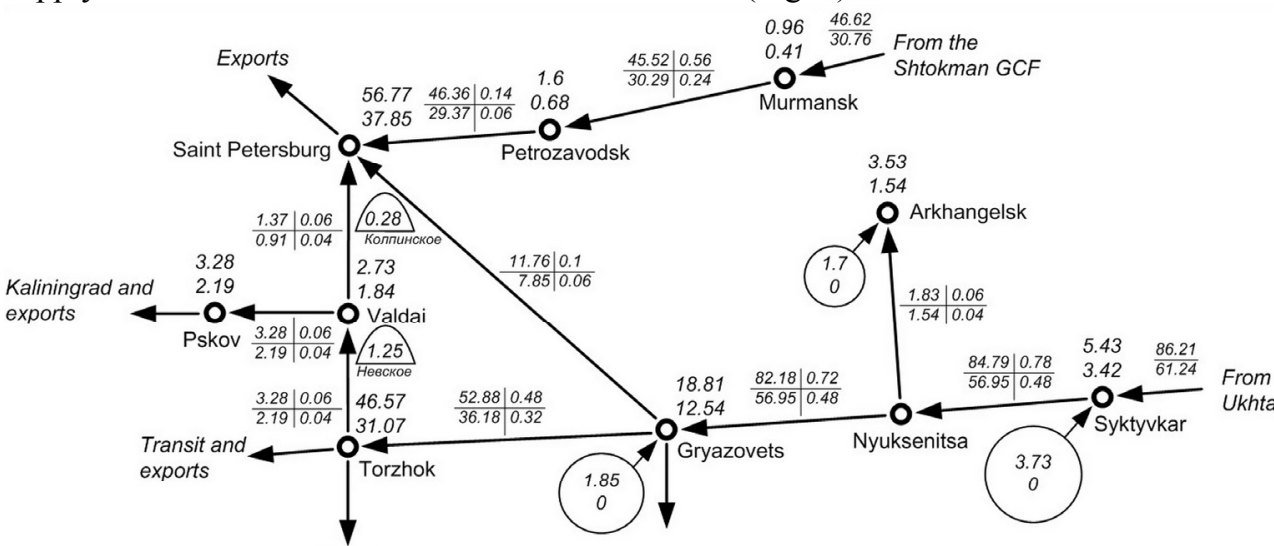

Transit

$$
\begin{aligned}
& \text { Working gas volume in a gas } \\
& \text { storage facility } \\
& \text { Gas demand at the node: } \\
& 0.96 \text { - winter season } \\
& 0.41 \text { - summer season } \\
& \text { O }
\end{aligned}
$$

Volume of gas transported and consumption of gas for internal needs:

Fig. 2. Regulation of seasonal irregularity of gas supply in the Northwestern Federal District, million tons of fuel equivalent 
This detailed problem-solving procedure is the main initial information for modeling the rational reliability of the GSS, which is carried out in two stages [2]:

Stage 1. Determine equivalent reliability characteristics (dependence of mathematical expectations of actual capacity and discounted costs on the installed performance).

Stage 2. Optimize the supply system redundancy:

$$
\begin{gathered}
\sum_{(i, j) \in U}\left(c_{i j} x_{i j}+k_{i j} y_{i j}\right)+p_{j} z_{j} \rightarrow \min \\
\sum_{i \in \Gamma_{j}^{+}}\left(\lambda_{i j} x_{i j}+\pi_{i j} y_{i j}\right)+\alpha_{j} z_{j}-\sum_{j \in \Gamma_{j}^{-}} x_{j i}=\left\{\begin{array}{l}
-Q, j=s ; \\
0, j \neq s, t ; \\
B, j=t .
\end{array}\right. \\
0 \leq x_{i j} \leq d_{i j} ; \quad 0 \leq y_{i j} \leq d_{i j}^{r}-d_{i j} ; 0 \leq z_{j} \leq Z_{j} .
\end{gathered}
$$

The minimum of the objective cost function is considered as a criterion, which takes into account the performance of facilities with existing redundancy $(\mathrm{x})$ and with additional reserve funds for these facilities $(y)$, as well as taking into account reserves of reserve fuel $(z)$.

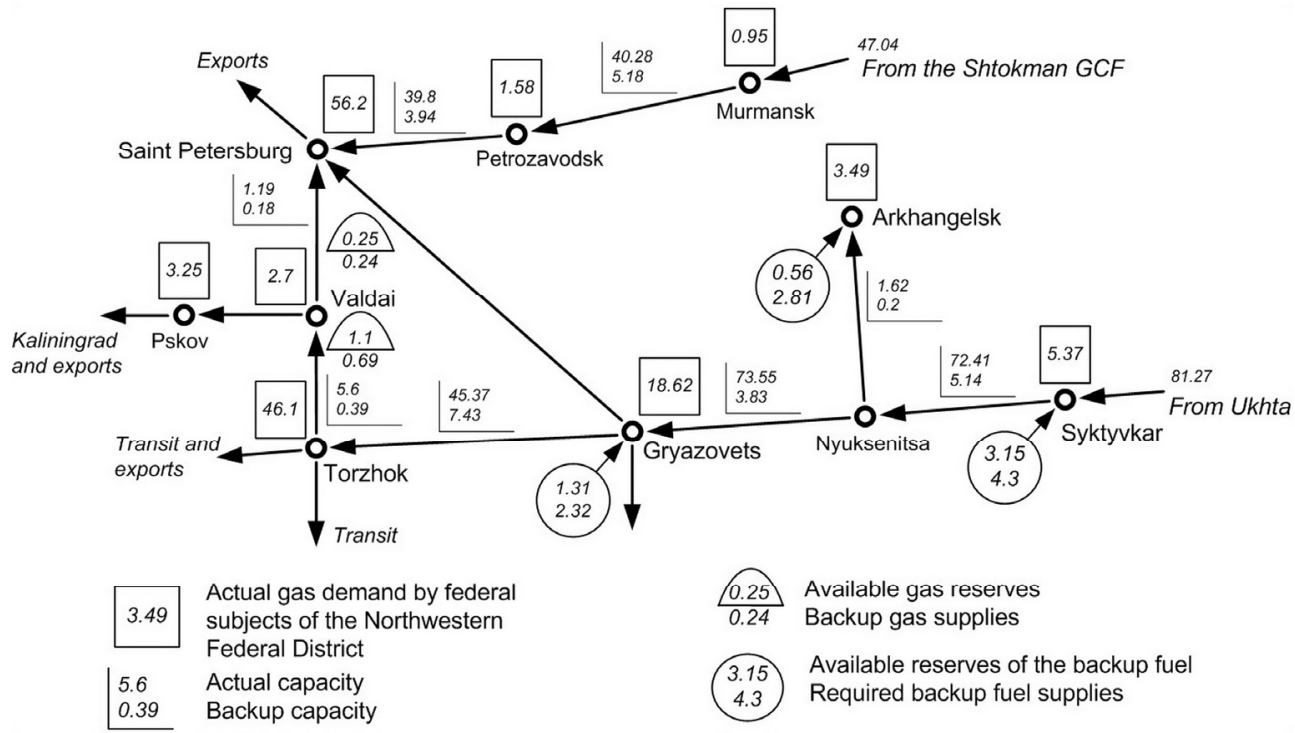

Fig. 3. Optimal supply redundancy of the gas supply system of the Northwestern Federal District in the winter period

Figure 3 shows the need to create a reserve capacity of the elements, as well as reserves of reserve fuel for some consumers in the district, to ensure the actual demand for gas in the constituent entities of the Northwestern Federal District with a supply ratio of 0.99 .

\section{Conclusion}

1. The modern development of gas supply systems, their renovation, and modernization require the consideration of the transformations in the mathematical models for their study.

2. Transformations of the mathematical model of flow distribution are considered. 
3. The proposed approaches are illustrated by calculating gas flows for the aggregated UGSS for the medium term, as well as by calculating the rational reliability of the Northwestern Federal District in the winter.

The research was carried out within the project III.17.4.3 of the Fundamental research program of SB RAS (AAAA-A17-117030310437-4)

\section{References}

1. Ed. by N.I.Voropai. Systems Research in the Energy Sector: Retrospective Scientific Directions of SEI-ESI (2010)

2. N.I. Ilkevich, T.V. Dzubina, Zh.V. Kalinina. Multilevel modeling of gas supply systems development (2014)

3. N.I. Voropai, B.G.Saneev., S.M. Senderov, et al. Energy of Russia in the XXI century. http://isem.irk.ru/energy 21 (2015)

4. L.R. Ford, D.R. Fulkerson. Flows in networks (1962)

5. M.G. Sukharev, E.R. Stavrovsky, E.E. Bryanskikh. Optimal development of gas supply systems (1981)

6. V.A. Yefremov Network flow models to control the operation and development of the unified gas supply system (1987)

7. M.G. Sukharev, E.R.Stavrovsky. Backing up trunk pipeline systems (1987)

8. A.P. Merenkov, E.V. Sennova, S.V. Sumarokov, et al. Mathematical modeling and optimization of heat, water, oil and gas systems (1992)

9. R. Madoliat, E. Khanmirza, A. Pourfard. Journal of Petroleum Science and Engineering. 149, P. 504 (2017).

10. A. Chebouba, F. Yalaoui, A. Smati, L. Amodeo, K. Younsi, A. Tairi. Computers \& Operations Research, 36 (6), 1916 (2009)

11. http://www.entsog.eu/public/uploads/files/publications/TYNDP/2015/entsog_TYNDP 2015_main_report_lowres.pdf (2015)

12. Ē. by V.A. Kulagin, T.A.Mitrova. Gas market in Europe: lost illusions and timid hopes (2015)

13. N. Christofides. Graph Theory. An Algorithmic Approach (1975)

14. N.I. Ilkevich, T.V. Dzyubina. The economy and mathematical methods, 38, 25 (2002).

15. E. Minieka Optimization algorithms for networks and graphs (1978)

16. Ed. by M.G. Sukharev. Reliability of gas and oil systems 1 (1994)

17. N.I. Ilkevich, W.I. Rabchuk, Y.D. Kononov. A Study of Methane Leakage in the Soviet Natural Gas Supply System, 30 (1991)

18. A.F. Dyakov, V.A. Stennikov, S.M. Senderov, etc. Reliability of energy systems (2014)

19. Energy Strategy of Russia for the period up to 2030, 48 (2009)

20. Zh.V. Kalinina. Development of methods for aggregation of gas supply systems (2016) 